\title{
CAN PROGRESSION OF ADOLESCENT IDIOPATHIC SCOLIOSIS BE STOPPED? A CASE SERIES CONSERVATIVE TREATMENT RESULTS
}

\author{
Bogdan GOGA ${ }^{1}$, Mariana CORDUN ${ }^{1}$ \\ ${ }^{1}$ National University of Physical Education and Sport, Faculty of Physical Therapy, Bucharest, Romania \\ *Corresponding author: bogdan.goga@ymail.com
}

https://doi.org/10.35189/dpeskj.2021.60.2.7

\begin{abstract}
Adolescent idiopathic scoliosis is the most common spinal deformity disorder. Although the current available literature presents a multitude of types of therapeutic interventions, data on their effectiveness are few. The research addresses three case studies observed in a short period of conservative treatment based on the Schroth method in order to highlight whether the Cobb angle progression and therefore scoliosis can be stopped during an evolutive period where the skeletal development is not finished. The Cobb angle was measured at the beginning on the most recent X-ray and after a period of six months on a new X-ray. Thus, the short-term effect of the therapeutic protocol was observed. The physiotherapy programme consisted of four Schroth-based exercises and an exercise to increase muscle strength and endurance, which were performed six times per week: three times in the rehabilitation clinic under the supervision of a Schroth physiotherapist, and three times the patient applied the therapy at home. Each session lasted about 60 minutes. Limitation may be due to the patient's coping strategies, the Cobb angle measurement or each participant's range of motion of the spine. Following these three case studies, we believe that the benefits of this approach can stop and correct the progression of adolescent idiopathic scoliosis, but further studies with a larger number of participants are needed.
\end{abstract}

Keywords: adolescent idiopathic scoliosis, Schroth method, Cobb angle.

\section{Introduction}

Scoliosis is a three-dimensional deformity of the spine and trunk, which may progress rapidly during periods of accelerated growth (Asher \& Burton, 2006; Goldberg et al., 2002).

Idiopathic scoliosis is a fixed lateral curvature of the spine greater than or at least equal to $10^{0}$, which affects about $2 \%$ to $3 \%$ of the entire population of children. It is this percentage that makes it the most common abnormality of the spine during the period of growth and development of a person (Lowe et al., 2000). Clinically, it is defined as a bone deformity with a subtle, painless onset in an apparently healthy subject. Although the female-to-male ratio is 1:1 for minor curves, this percentage increases dramatically in the female gender as the severity of the curves increases (Weinstein et al., 2008). The cause of adolescent idiopathic scoliosis is not known so far; however, some theories bring into discussion the neurological and hormonal implications of this condition (Wang et al., 2011). Instead, despite widespread opinions, it is known that the poor posture pattern cannot explain the onset of this pathology and can only be considered an aggravating factor.

Most patients with scoliosis ( $80 \%$ to $90 \%$ ) have an insufficient cause and are diagnosed with idiopathic scoliosis. In other cases, scoliosis is secondary to other pathologies such as neuroborreliosis, cerebral palsy, congenital abnormalities of vertebral development, neoplastic formations or various syndromes (e.g., Marfan). The treatment of symptomatic scoliosis will 
be influenced by its cause. As scoliosis develops during growth spurts but also in adulthood, the main goal of the therapeutic intervention is to stop the progression of the curve (Weiss \& Moramarco, 2013).

Being a condition that produces three-dimensional deformations, it is worth mentioning that the spine has a lateral deviation in the frontal plane on a sagittal axis, which will lead to the formation of a convexity and a concavity. In the sagittal plane, the curves most often decrease and thoracic hypokyphosis (which, in severe cases, can be reversed into lordosis) associated with lumbar hypolordosis is noticed, while in the transverse plane, there is vertebral rotation on a frontal axis especially at the apex of the curve, which occurs on the same side as the convexity.

Schroth therapy is a physiotherapeutic method for the treatment of idiopathic scoliosis, which has shown promising results in several studies (Park et. al., 2018). The concept focuses on developing the corrective posture reflex integrated into daily activities: treatment of the entire spine rather than just the scoliotic curvature; static and dynamic modification of the projection of the centre of gravity; training to develop muscle strength and endurance in an antigravity posture; patient education on a better understanding of deformity in order to increase compliance with conservative treatment.

In classic physiotherapy treatment, concepts are used in which the convexity area is considered to be hypotonic and the concavity area, hypertonic. Thus, classic physiotherapy has as objectives the development of muscle tone on the hypotonic side and its relaxation on the hypertonic side. This concept can no longer be relevant, an aspect observed in both the Schroth concept and our personal practice. There are cases in which, following the musculoskeletal assessment, the patient's generalised hypotonia associated with increased joint mobility is observed or cases in which muscle tone is increased and range of motion is diminished. Moreover, muscle tone is a muscle characteristic regulated by the central nervous system, and therapeutic exercise can indirectly influence it. Most classic exercises do not aim to correct or direct the projection of the centre of gravity to influence the spatial position of the trunk and have a working position parallel to gravity. Instead, the goal desired by both the patient, their family and the multidisciplinary team is to correct the curvature. This goal can be unrealistic in many situations, especially in a period of accelerated patient growth in which skeletal development has not been reached.

The aim of these case studies is to highlight the role of physiotherapy based on Schroth principles in the treatment of adolescent idiopathic scoliosis for patients aged 12 to 14 years and create a preliminary basis for further and more extensive research.

The research objective is to highlight the role of physiotherapy in the multimodal treatment of adolescent idiopathic scoliosis.

Research question: Can conservative treatment of idiopathic scoliosis by physiotherapy based on Schroth principles stagnate the development of scoliosis highlighted by the progression factor? 


\section{Methodology}

\section{Participants}

The three case studies consisted of patients who received the recommendation of physiotherapy from the orthopaedic paediatrician and requested this service at the Medical Rehabilitation Clinic "Terapie pentru mișcare" ("Therapy for movement") between July 2018 and August 2019.

The inclusion criteria were:

- Medical diagnosis of adolescent idiopathic scoliosis;

- Risser sign no more than 1 (0 and 1);

- Cobb angle more than $30^{\circ}$ in at least one curvature;

- Risk of progression more than $90 \%$.

The exclusion criteria were:

- Scoliosis of known cause (abnormal congenital vertebrae, cerebral palsy, different syndromes);

- Risser sign more than 1 (2, 3, 4 or 5);

- Cobb angle less than $30^{\circ}$ in current curvatures;

- Risk of progression less than $90 \%$.

\section{Instruments}

The assessment of scoliosis begins with the clinical examination, after which the orthopaedic paediatrician may suspect this diagnosis. For confirmation, the specialist will request an X-ray examination that will confirm or refute the suspicion of diagnosis. In addition, this investigation is important to both determine the type of treatment, which can be either conservative or surgical, and design a physiotherapy programme based on Schroth principles.

In 1948, Dr John Cobb describes a technique for measuring the magnitude of scoliosis in the frontal plane (Lonner et al., 2010). The angle of curvature will be measured by drawing, in a first phase, two lines parallel to the vertebral plate of each neutral vertebra. Starting from these lines of the vertebral plate, two perpendiculars will be drawn - one from each vertebral plate to the other vertebra. The angle formed by the intersection of these perpendiculars represents the Cobb angle (Cordun, 2009). There is an accepted calculation error between 3 and $5^{0}$. For this reason, a "real difference" is considered a change of more than $5^{0}$ (Lonner et al., 2010). If properly measured, it can provide accurate information about the progression of a curve, the effectiveness of orthopaedic and rehabilitation treatment, the results of surgery and maintaining the correction over time. Furthermore, the type of (conservative or surgical) treatment will be indicated based on Stagnara's criteria for assessing the angle.

The Risser sign is a radiological sign often used to define skeletal development, especially in the treatment of idiopathic scoliosis. The sign can be observed on the frontal incidence of the radiographic examination of the iliac crest. The fact that the X-ray will also include the pelvis makes this sign preferable to other signs of skeletal maturation (olecranon apophysis or degree of bone maturation of the hand), given that it will not require another radiographic exam 
and will limit the level of radiation to which the patient is exposed. The Risser sign is divided into six stages from 0 to 5, with stage 0 corresponding to two thirds of the accelerated pubertal growth phase (Akbarnia et al., 2010). Bone age and skeletal development will be framed in a clinical context along with chronological age, annual height increase and sexual development characteristics in order to determine more accurately the therapeutic options for the patient with scoliosis (Roberto et al., 1997).

The cause of idiopathic scoliosis is not known or why some curves worsen and others do not. Thus, calculating the progression index of a curve can be very useful in clinical practice to have a more realistic picture of the patient's condition and to set realistic goals closer to each one's characteristics. It is important for the specialist to understand the relationship between growth potential and the risk of progression when considering treatment options, especially in patients at high risk of progression (Weiss et al., 2018). This risk can be calculated using the formula described by Lonstein and Carlson (1984), which is still accepted by most specialists treating this disease. The International Society on Scoliosis Orthopaedic and Rehabilitation Treatment (SOSORT) developed guidelines derived from this formula to avoid over or under treatment (Weiss et al., 2018). Lonstein and Carlson's formula is:

$$
\frac{\text { Cobb angle }-(3 \times \text { Risser sign })}{\text { cronological age }}
$$

The result of the formula is a progression factor that will be correlated with the graph described by the two authors (Lonstein \& Carlson, 1984) to identify the incidence of progression (Figure 1).

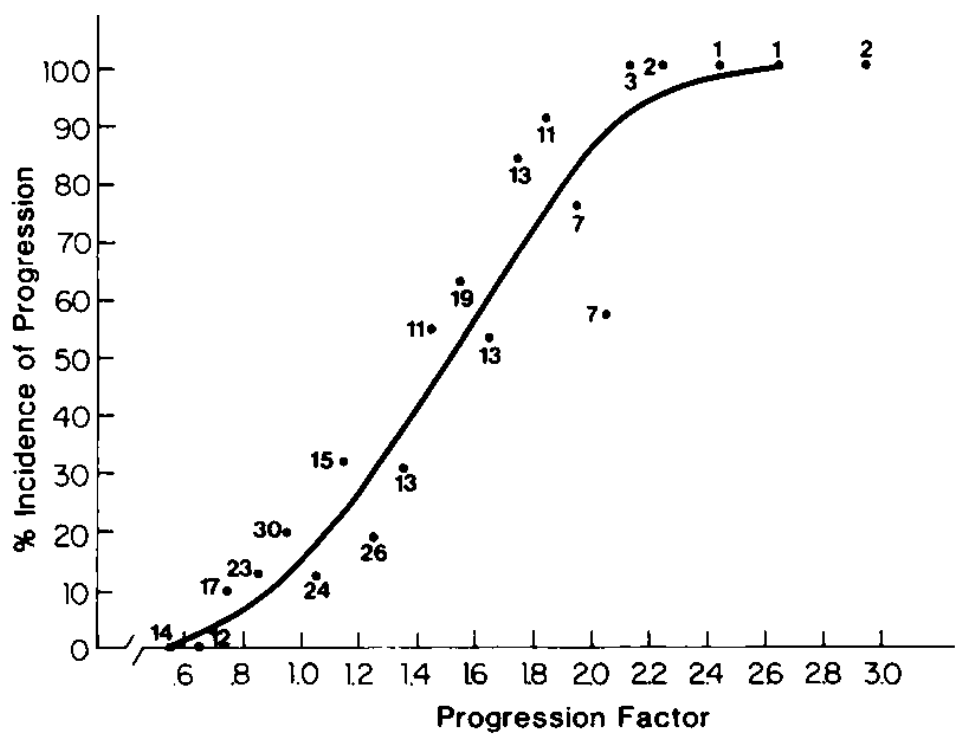

Figure 1. Calculation of the scoliosis incidence of progression

During childhood and adolescence, there are periods of accelerated growth in which the curvature is very likely to worsen. In periods of slow growth, worsening is unlikely (Hawes \& O'Brien, 2006). The more immature it is in terms of bone maturation, the greater the risk of curvature progression (Weinstein et al., 2003). In the same way, the greater the curvature at diagnosis, the greater its risk of progression (Lowe et al., 2000). 


\section{Procedure}

The proposed programme consists of three sessions per week for six months, which are performed in the clinic under the supervision of a physiotherapist specialising in Schroth therapy, and three sessions performed at home where the patient is initially supervised by a parent until they are able to reach the level at which they can perform the programme without supervision. The duration of each session is approximatively 60 minutes.

Conservative treatment also included the use of a Chêneau-type brace according to Stagnara's criteria. The recommendation was to wear the brace for approximatively 22-23 hours. This type of medical device has the same principles as the Schroth method, creating breathing expansion zones in the trunk area, which are referred to as "breathing zones" in the Schroth method. Moreover, the brace exerts a three-dimensional pressure on the anterior and posterior gibbosities, which is similar to the types of mobilisation techniques and corrections used in the German physiotherapy method.

Compliance with and acceptance of the physiotherapy programme as well as wearing the brace are important issues and, if they are at a low level, the physiotherapist has the responsibility to discuss with the family, physician and psychotherapist to find a way together to make therapeutic programmes more tolerable and increase patient involvement.

In the Schroth-based physiotherapy, most exercises (except those in ventral and dorsal decubitus) will be performed in front of a mirror to constantly integrate visual feedback through which the patient becomes aware of and then learns the corrective and hypercorrective posture. In some cases, mirrors positioned posteriorly and laterally by the patient can be used to improve their spatial orientation and provide three-dimensional visual feedback as complex as possible.

From the first sessions, the aim is to educate the patient on the projection of the centre of gravity in both orthostatism, sitting or other positions used in daily activities. Thus, a first step is to train the patient's awareness of the preferential loading of a lower limb, which is generally the lower limb adjacent to the convexity of the main curve.

Another aspect that is pursued during exercise is the education of respiratory function. The patient is instructed to remain active while inhaling and exhaling by active isometric muscle contraction in order to maintain the areas of anterior and posterior gibbosities in a position of maximum expiration and expand the anterior and posterior depressive areas in a position of maximum inspiration. The challenge will be to maintain the isometric expansion of the depressions, which are referred to as "breathing zones" in this concept, in both the inspiratory phase and the expiratory phase by isometric contraction. Thus, the Schroth therapy concept describes this type of breathing as angular breathing that is performed on the diagonal of the depressions and stagnant, as far as possible, on the diagonal of the gibbosities. Moreover, the aim is for the expiratory phase to be as long as possible, and for the inspiratory phase to be performed in a relaxed way, with a normal volume of air. To facilitate the expiratory phase, the patient is instructed to exhale into the oral cavity by pronouncing the letter " $\mathrm{S}$ ".

Once the patient has mastered the concepts of projection of the centre of gravity and guided breathing and manages to reach a corrective position, the physiotherapist can begin to design a therapeutic exercise that will include all three elements plus basic muscle tension. In the expiratory phase, the isometric contraction of the whole body will be accomplished. The exercise will be performed in a fundamental posture with corrective positioning that will be 
maintained isometrically for 4-5 respiratory cycles up to 10 respiratory cycles. Patients who have experience with this therapy can even reach the performance of doing the exercise for 5 minutes without a break. The programme will consist of 5 exercises. The difference between exercises is the fundamental posture used by the patient, the other aspects specific to Schroth therapy (basic tension, generalised isometry, angular breathing, projection of the centre of gravity, visual, tactile and auditory feedback) being performed in the same way.

The proposed physiotherapy programme based on the principles described by Katharina Schroth consists of four Schroth therapy exercises and an exercise to increase muscle strength and endurance.

- Exercise 1: Symmetric and asymmetric muscle cylinder from standing - 4 sets of 10 respiratory cycles. The asymmetric position will be created by loading the leg on the side of the lumbar convexity, which is called "L-side" in the Schroth concept. The other leg will be placed on a chair with the knee extended. The therapist will choose the height according to the Schroth functional diagnosis and the patient's height.

- Exercise 2: Side-lying muscle cylinder in front of a mirror - 4 sets of 10 respiratory cycles. This exercise will be always done on the side of the lumbar curve (opposite to the thoracic convexity)

- Exercise 3: Big Bow performed at the gym wall bars - 4 sets of 10 respiratory cycles. This type of exercise will increase the self-elongation of the patient's spine.

- Exercise 4: Standing cylinder using two Schroth gymnastic sticks - 4 sets of 10 respiratory cycles. The grip will be symmetric or asymmetric according to the traction-countertraction principles described in the German method.

- Exercise 5: Low (forearm) plank - 2 sets of 1 minute. The patient will be instructed to maintain all Schroth-specific corrections during this isometric exercise.

\section{Results}

The case studies included three female participants who were diagnosed with adolescent idiopathic scoliosis under one month. The Chêneau-type brace was the first medical device that all three cases received for their pathology. Their medical histories allowed the centralisation of personal information: patients' first and last names, age, gender, diagnosis and compliance with the Risser sign as well as calculation of the Cobb angle and progression index (Table 1).

Regarding the implementation of the proposed physiotherapy programme, the case studies meet the inclusion criteria and do not match the exclusion criteria.

Table 1. Personal information on the investigated participants

\begin{tabular}{lccccc}
\hline Case & Gender & Age & Diagnosis & $\begin{array}{c}\text { Initial Cobb } \\
\text { angle }\end{array}$ & $\begin{array}{c}\text { Incidence of } \\
\text { progression }\end{array}$ \\
\hline 1 & $\mathrm{~F}$ & 12 & Adolescent idiopathic scoliosis & $41^{\circ}$ & $100 \%$ \\
2 & $\mathrm{~F}$ & 12 & Adolescent idiopathic scoliosis & $37^{\circ}$ & $100 \%$ \\
3 & $\mathrm{~F}$ & 12 & Adolescent idiopathic scoliosis & $41^{\circ}$ & $100 \%$ \\
\hline
\end{tabular}




\section{Case 1: A.S. - Initial assessment}

12-year-old female patient; diagnosis of idiopathic thoracolumbar scoliosis, right convexity, adolescent idiopathic scoliosis; Cobb angle of $41^{\circ}$, with the upper neutral vertebrae at the level of the thoracic vertebrae 10 and the lower neutral vertebrae at the level of the lumbar vertebrae 3. Functional diagnosis according to Schroth classification: LriHleKt (lumbar right, pelvis left, hypokyphosis); the calculated progression factor of the curvature is 3.16 , which indicates a $100 \%$ incidence of scoliotic curvature progression. The Risser sign was 0.

Assessment after six months - the Cobb angle measurement reveals a curvature of $26^{0}$, with a decrease of $15^{0}$ compared to the initial assessment where the upper neutral vertebrae were at the level of the thoracic vertebrae 10 and the lower neutral vertebrae, at the level of the lumbar vertebrae 3. The Risser sign was 1.

Case 2: T.S. - Initial assessment

12-year-old female patient; diagnosis of idiopathic thoracic scoliosis, right convexity, adolescent idiopathic scoliosis; Cobb angle of $37^{\circ}$, with the upper neutral vertebrae at the level of the thoracic vertebrae 7 and the lower neutral vertebrae at the level of the thoracic vertebrae 12. Functional diagnosis according to Schroth classification: TriHle (thoracic right, pelvis left); the calculated progression factor of the curvature is 3.08, which indicates a $100 \%$ incidence of scoliotic curvature progression. The Risser sign was 0 .

Assessment after six months - the Cobb angle measurement reveals a curvature of $27^{0}$, with a decrease of $10^{\circ}$ compared to the initial assessment where the upper neutral vertebrae were at the level of the thoracic vertebrae 7 and the lower neutral vertebrae, at the level of the lumbar vertebrae 12. The Risser sign was 1.

\section{Case 3: B.P. - Initial assessment}

12-year-old female patient; diagnosis of idiopathic thoracolumbar scoliosis, left convexity, adolescent idiopathic scoliosis; Cobb angle of $41^{\circ}$, with the upper neutral vertebrae at the level of the thoracic vertebrae 10 and the lower neutral vertebrae at the level of the lumbar vertebrae 4. Functional diagnosis according to Schroth classification: LleHriKt (thoracic right, pelvis left); the calculated progression factor of the curvature is 4.1 , which indicates a $100 \%$ incidence of scoliotic curvature progression. The Risser sign was 0 .

Assessment after six months - the Cobb angle measurement reveals a curvature of $26^{0}$, with a decrease of $15^{0}$ compared to the initial assessment where the upper neutral vertebrae were at the level of the thoracic vertebrae 10 and the lower neutral vertebrae, at the level of the lumbar vertebrae 4 . The Risser sign was 1.

In all three cases, a decrease of at least $5^{0}$ is observed. Therefore, it cannot be considered a measurement error/variation (differences below $5^{0}$ ). The average of the studied group is $13.33^{0}$ Cobb angle correction. (Table 2 and Figure 2)

Table 2. Changes in the Cobb angle between initial assessment and after six months

\begin{tabular}{lcccc}
\hline \multirow{2}{*}{ Item no. } & \multirow{2}{*}{ Cases } & \multicolumn{3}{c}{ Cobb angle } \\
\cline { 3 - 5 } & & Initial & After six months & Differences \\
\hline 1 & A.S. & $41^{0}$ & $26^{0}$ & $15^{0}$ \\
2 & T.S. & $37^{0}$ & $27^{0}$ & $10^{0}$ \\
3 & B.P. & $41^{0}$ & $26^{0}$ & $15^{0}$ \\
& & 39.66 & 26.33 & 13.33 \\
\hline
\end{tabular}




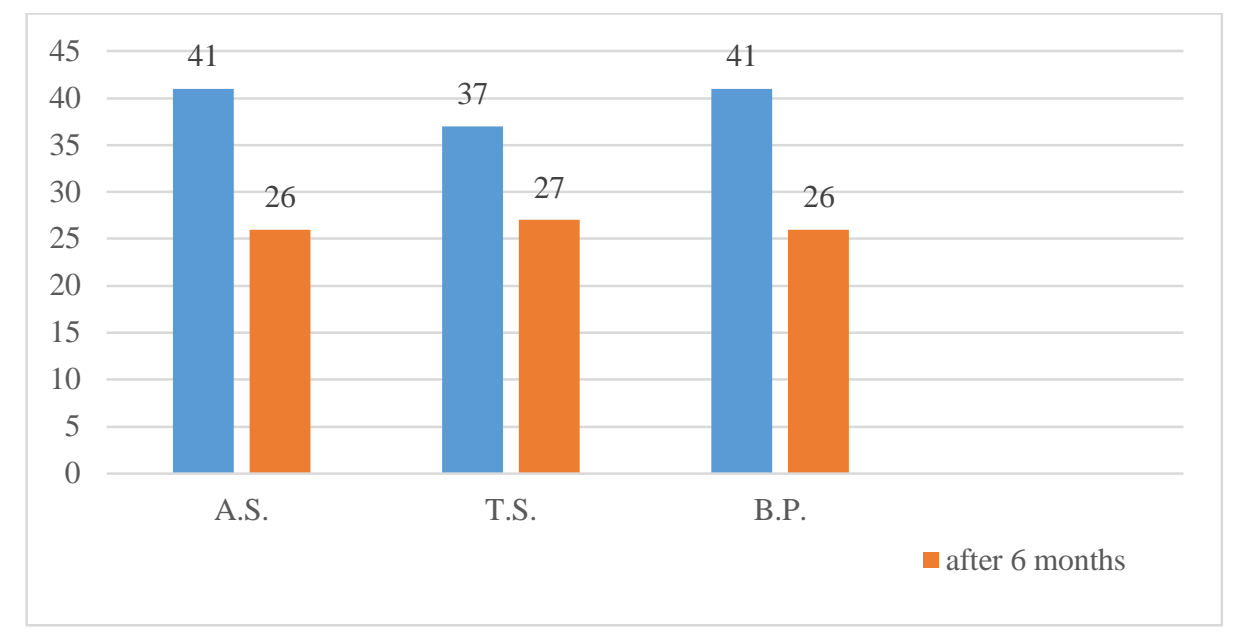

Figure 2. Graphical representation of the Cobb angle (initial assessment and after six months)

A greater decrease is observed in cases 1 (A.S.) and 3 (B.P.) compared to case 2 (T.S.). A notable difference between cases is that, in case 1 and case 3 , the main curvature is lumbar, while for T.S., the main curvature is thoracic. In all three cases, it is noticed that the value of the Cobb angle decreases by more than $5^{\circ}$ in the analysed curves, which reveals not only that the progression of cases has been stopped but also their correction. Thus, the effectiveness of the proposed programme can be the basis for further research.

\section{Conclusion}

The case studies associated Schroth therapy with wearing a Chêneau brace. This therapeutic approach complies with Stagnara's criteria for the treatment of idiopathic scoliosis. Given that the corrective and hypercorrective effect of the Chêneau brace is well known, the discussion arises as to how long before getting an X-ray the brace should be removed in order to have a more objective examination and eliminate its postural effect. There is no international consensus on this, the recommendation being made with four hours before the X-ray, from our personal experience.

In all of three case studies, patients had good active range of motion of the spine, which was measured at the initial assessment and after six months. The results of the physiotherapy programme can be influenced by this parameter. Therefore, for a broader analysis of the effectiveness of the Schroth method, a future comparative study is proposed between a population with good spinal mobility and muscle flexibility and a population with spinal rigidity.

All three cases had in common that they showed a high level of compliance with and involvement in the therapeutic plan. We believe that this is a key aspect of the therapeutic success and points out the need for a coping strategy in patients with a low level of involvement.

The study of the literature has shown that the progression of idiopathic scoliosis cannot be stopped with conservative treatment, especially if the incidence progression is around $100 \%$ (Goldberg et al., 1995). The results obtained in the three case studies based on the specific physiotherapy programme using the Schroth method show the opposite, all cases indicating a 
significant clinical correction (greater than $5^{\circ}$ Cobb angle) at the end of the six months of physiotherapy.

These results allow us to continue by extending the application of the programme to a larger number of participants, in the hope of obtaining clearer conclusions about the importance of systematically applying physiotherapy based on the principles of Katharina Schroth to patients with adolescent idiopathic scoliosis. Further studies on the long-term effects of conservative treatment are also recommended and represent one of the authors' future plans.

\section{References}

Akbarnia, B. A., Yazici, M., \& Thompson, G. H. (2010). The growing spine management of spinal disorders in young children. Springer.

Asher, M. A., \& Burton, D. C. (2006). Adolescent idiopathic scoliosis: Natural history and long-term treatment effects. Scoliosis, 1(1): 2. https://doi.org/10.1186/1748-7161-1-2

Cordun, M. (2009). Kinantropometrie [Kinanthropometry]. CD Press.

Goldberg, C. J., Dowling, F. E., Fogarty, E. E., \& Moore, D. P. (1995). School scoliosis screening and the United States Preventive Services Task Force. An examination of longterm results. Spine, 20(12), 1368-1374. PMID: 7676334

Goldberg, C. J., Moore, D. P., Fogarty, E. E., \& Dowling, F. E. (2002). Adolescent idiopathic scoliosis: Natural history and prognosis. Studies in Health Technology and Informatics, 91, 59-63. PMID: 15457694

Hawes, M. C., \& O'Brien, J. P. (2006). The transformation of spinal curvature into spinal deformity: Pathological processes and implications for treatment. Scoliosis, 1(1): 3. https://doi.org/10.1186/1748-7161-1-3

Lonner, B. S., Auerbach, J. D., Sponseller, P., Rajadhyaksha, A. D., \& Newton, P. O. (2010). Variations in pelvic and other sagittal spinal parameters as a function of race in adolescent idiopathic scoliosis. Spine, 35(10), E374-E377. https://doi.org/10.1097/brs.0b013e3181bb4f96

Lonstein, J. E., \& Carlson J. M. (1984). The prediction of curve progression in untreated idiopathic scoliosis during growth. The Journal of Bone and Joint Surgery. American volume, 66(7), 1061-1071. https://doi.org/10.2106/00004623-198466070-00013

Lowe, T. G., Edgar, M., Margulies, J. Y., Miller, N. H., Raso, V. J., Reinker, K. A., Rivard, C. H. (2000). Etiology of idiopathic scoliosis: Current trends in research. The Journal of Bone and Joint Surgery, 82(8), 1157-1168. https://doi.org/10.2106/00004623-200008000-00014

Park, J.-H., Jeon, H.-S., \& Park, H.-W. (2018). Effects of the Schroth exercise on idiopathic scoliosis: A meta-analysis. European Journal Physical and Rehabilitation Medicine, 54(3), 440-449. https://doi.org/10.23736/s1973-9087.17.04461-6

Roberto, R. F., Lonstein, J. E., Winter, R. B., \& Denis, F. (1997). Curve progression in Risser stage 0 on patients after posterior spinal fusion for idiopathic scoliosis. Journal of Pediatric Orthopaedics, 17(6), 718-725. https://doi.org/10.1097/00004694-199711000-00005

Wang, W. P., Yeung, H. Y., Chu, W. C.-W., Tang, N. L.-S., Lee, K. M., Qiu, Y., Burwell, R. G., \& Cheng, J. C. Y. (2011). Top theories for the etiopathogenesis of adolescent idiopathic scoliosis. Journal of Pediatric Orthopaedics, 31(1 Suppl), S14-S27. https://doi.org/10.1097/bpo.0b013e3181f73c12

Weinstein, S. L., Dolan, L. A., Cheng, J. C. Y., Danielsson, A., \& Morcuende, J. A. (2008). Adolescent idiopathic scoliosis. The Lancet, 371(9623), 1527-1537. https://doi.org/10.1016/S0140-6736(08)60658-3 
Weinstein, S. L., Dolan, L. A., Spratt, K. F., Peterson, K. K., Spoonamore, M. J., \& Ponseti, I. V. (2003). Health and function of patients with untreated idiopathic scoliosis: A 50-year natural history study. JAMA, 289(5), 559-567. https://doi.org/10.1001/jama.289.5.559

Weiss, H. R., \& Moramarco, M. (2013). Scoliosis - treatment indications according to current evidence. Musculoskeletal Medicine, 1(1): 1. http://dx.doi.org/10.13172/2052-9287-1-1-347

Weiss, H. R., Lehnert-Schroth, C., Moramarco, M., \& Moramarco, K. (2018). Schroth therapy: Advancements in conservative scoliosis treatment (2nd ed.). Lap Lambert Academic Publishing. 